\title{
Leg Wound Complications: A Comparison Between Endoscopic and Open Saphenous Vein Harvesting Techniques
}

\author{
Yasser Mubarak, ${ }^{1,2}$ Ahmed Abdeljawad ${ }^{3,4}$ \\ ${ }^{1}$ Cardiothoracic Surgery Department, Minia University, Egypt; \\ ${ }^{2}$ Madinah Cardiac Center, KSA; \\ ${ }^{3}$ Cardiothoracic Surgery Department, Cairo University, Egypt; \\ ${ }^{4}$ Clinical of Cardiothoracic \& Vascular Surgery, Städtisches Klinikum Braunschweig, Germany
}

\section{ABSTRACT}

Background: The most common conduit for coronary artery bypass graft (CABG) surgery is saphenous vein graft (SVG). There are two techniques for SVG harvesting: open and endoscopic. Our aim is to evaluate clinical results of endoscopic versus open SVG harvesting. Nowadays, endoscopic vein harvesting $(\mathrm{EVH})$ has become prevalent because of reduced complications with more patient satisfaction.

Objective: We designed and performed a prospective randomized cohort study of patients undergoing CABG to compare the results of open versus endoscopic harvesting technique.

Methods: Patients who underwent elective CABG at our hospitals were divided into two groups, during the period of January 2019 to March 2021. The EVH group (50 patients) underwent endoscopic technique compared with the open vein harvesting $(\mathrm{OVH})$ group (50 patients) that was underwent open surgical incision for great saphenous vein (GSV) harvesting. The two groups demographically were similar and received identical management. Leg wound was evaluated at discharge, two weeks, and four weeks for evidence of any complications. Early outcomes were recorded, including infection, gaped wound and surgical re-suture, degree of pain, level of cosmetic satisfaction, and early mobilization.

Results: In the EVH group, harvesting time increased, and incision closure time decreased in comparison with $\mathrm{OVH}$. The hospital stay was $5.5 \pm 2.4$ days in the EVH group versus $9.5 \pm 2.7$ days in the OVH group. Leg wound complications were significantly reduced in the EVH group in comparison with the OVH group.

Conclusions: Endoscopic vein harvesting technique reduced leg wound complications. Conveniently, patients also were cosmetically satisfied.

Received April 24, 2021; accepted May 20, 2021.

Correspondence: Yasser Shaban Mohamed Mubarak, Assistant Professor, Cardiothoracic Surgery Department, Faculty of Medicine, Minia University, ElMinya, 61111, Egypt, Telephone +966560708223, +201002554078 (e-mail: yassermubarak73@gmail.com).

\section{INTRODUCTION}

Coronary artery bypass grafting (CABG) surgery remains the most common procedure in adult cardiac surgery for coronary artery disease (CAD). Saphenous vein grafting (SVG) remains the most commonly used bypass conduit in CABG because it is usually available at desired length, large diameter, easily harvested, and does not affect sternal wound healing [Moshkovitz 2016].

Open vein harvesting $(\mathrm{OVH})$ technique is a linear incision along the course of GSV under direct vision. However, $\mathrm{OVH}$ technique carries an increased risk of wound complications, including infection, hematoma, seroma, pain, and mobility limitation. Leg wound complications include prolonged wound healing, hospital stay, and poor cosmetic outcome. It may require readmission for debridement or IV antibiotics [Raghuram 2007]. EVH is a less invasive technique and associated with $2 \%$ to $5 \%$ incidence of wound complications. It reduces postoperative pain, small leg incisions, and increases patient satisfaction [Kalra 2016]. OVH technique has an incidence of $3 \%$ to $30 \%$ wound complication. It may necessitate extending the incision above the knee with more complications [Mandiye 2007]. Since 1996, the first clinical report of $\mathrm{EVH}$ was published; it increased in popularity to become the preferred method of SVG harvesting [Zenati 2011]. EVH is an atraumatic procedure that harvests SVG without direct manipulation of the vein [Raza 2018]. EVH is usually done from the thigh, however the conduit is usually a short length. The endoscope could be negotiated through the same entry wound at knee level to harvest an additional length from the leg without conversion to open technique [Mandiye 2007].

\section{METHODS}

A prospective, random group trial was undertaken between January 2019 to March 2021 to compare EVH with $\mathrm{OVH}$ in patients admitted for elective CABG. Patients were divided into the EVH group $(N=50)$ and OVH group $(N=$ 50). Informed consent was taken for every case. We excluded cases that were converted to $\mathrm{OVH}$ and those with combined technique (both $\mathrm{EVH}$ and $\mathrm{OVH}$ ).

GSV was exposed by means of a $2 \mathrm{~cm}$ transverse incision along the medial surface of the knee. The vein was dissected 


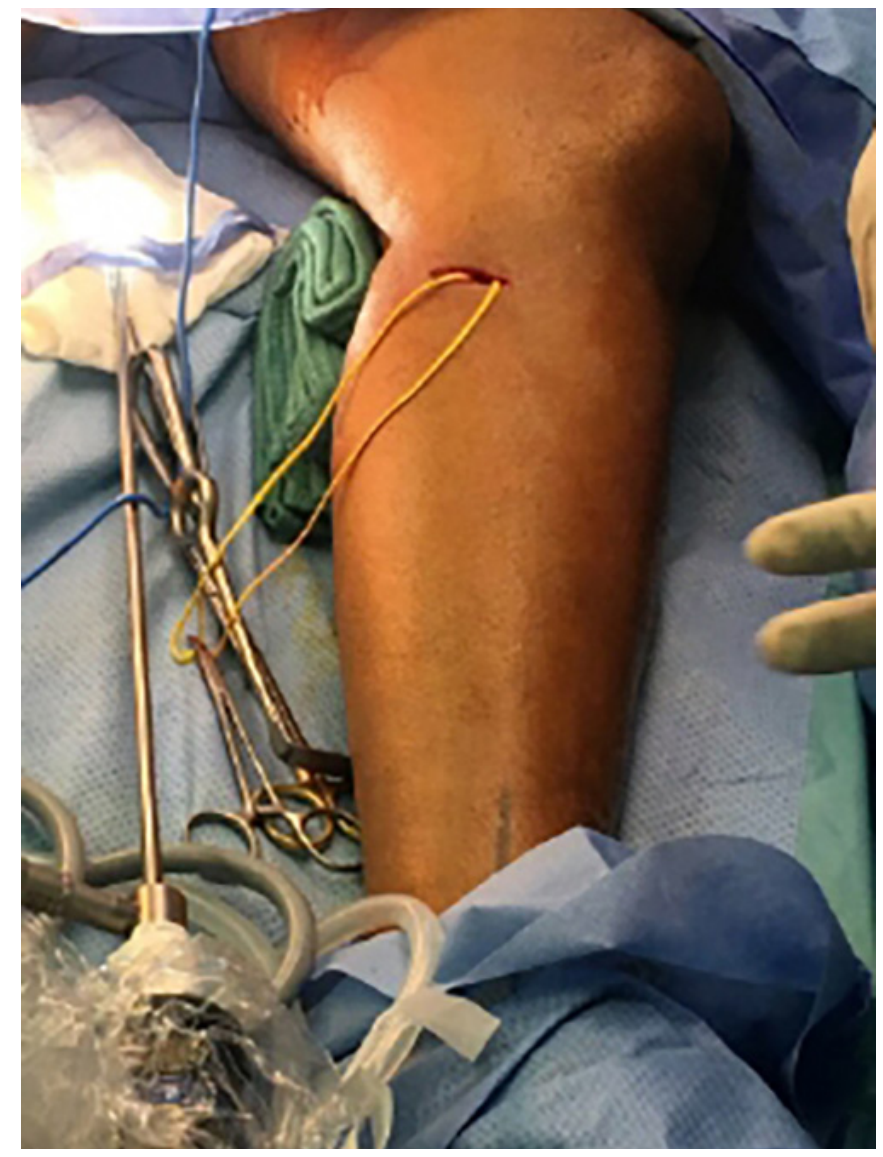

Figure 1. EVH through small incision below knee free and surrounded by a vessel loop. Subcutaneous tunnels were created distally and proximally. The endoscopic dissecting device VirtuoSaph @Plus was placed in proximal space (toward the groin). A tunnel was created by blunt dissection along the length of the saphenous vein in the thigh. After 3-5 cm of blunt dissection, insufflation was performed by using carbon dioxide (pressure of $13 \mathrm{mmHg}$ and flow $4 \mathrm{~L} / \mathrm{min}$ ). We did not use an insufflation port to avoid incidence of $\mathrm{CO} 2$ embolism. Heparin was given to reduce incidence of clot formation. Sometimes, we did not give heparin before harvesting to avoid excess bleeding during

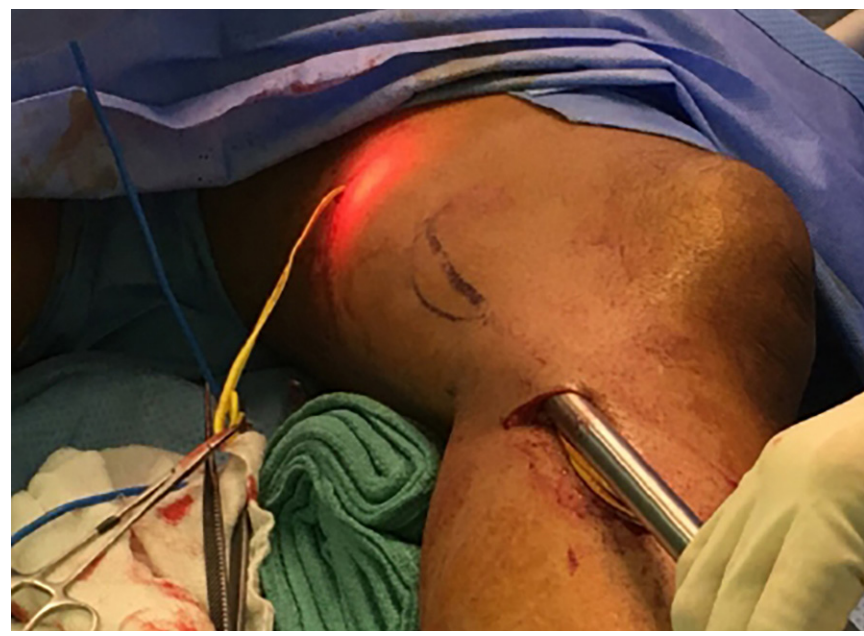

Figure 2. Dissection of GSV through two small incisions below knee and thigh
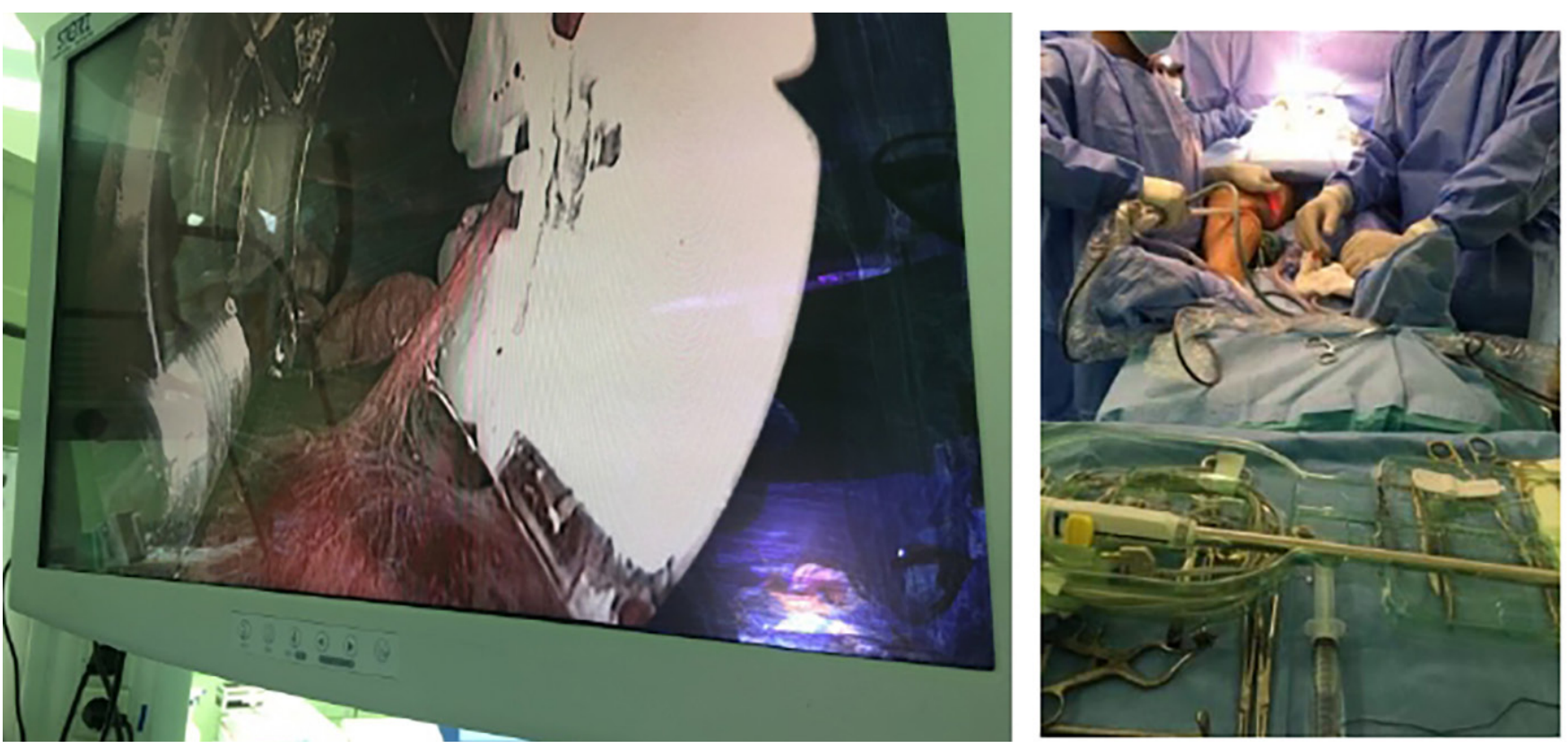

Figure 3. Coagulation of GSV tributaries by EVH 
mammary harvesting. The vein was circumferentially dissected with its tributaries by using a C-ring dissector and bipolar loop scissors (dissector cautery device). Once all tributaries were divided, $0.5 \mathrm{~cm}$ incision was made at the groin and the proximal end of the vein was divided. SVG was harvested from the thigh then leg, if more length was needed; the same entry point of incision can be used as in

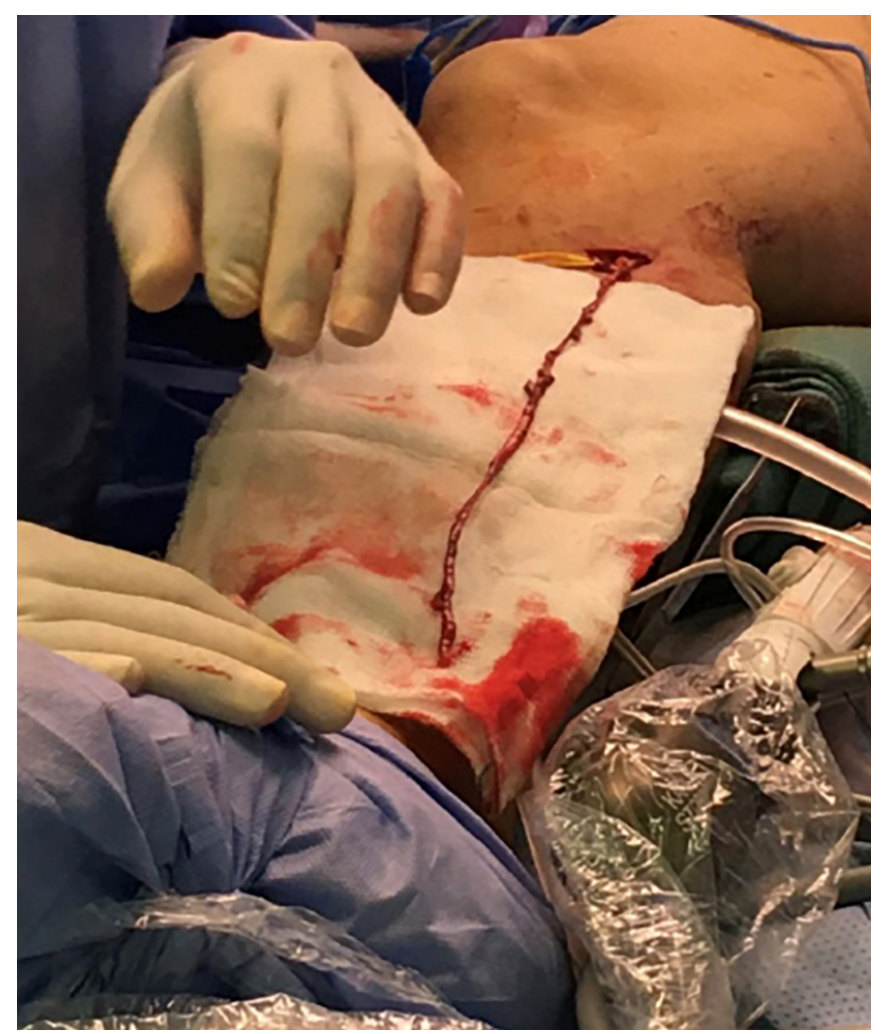

Figure 4. Harvesting GSV endoscopically

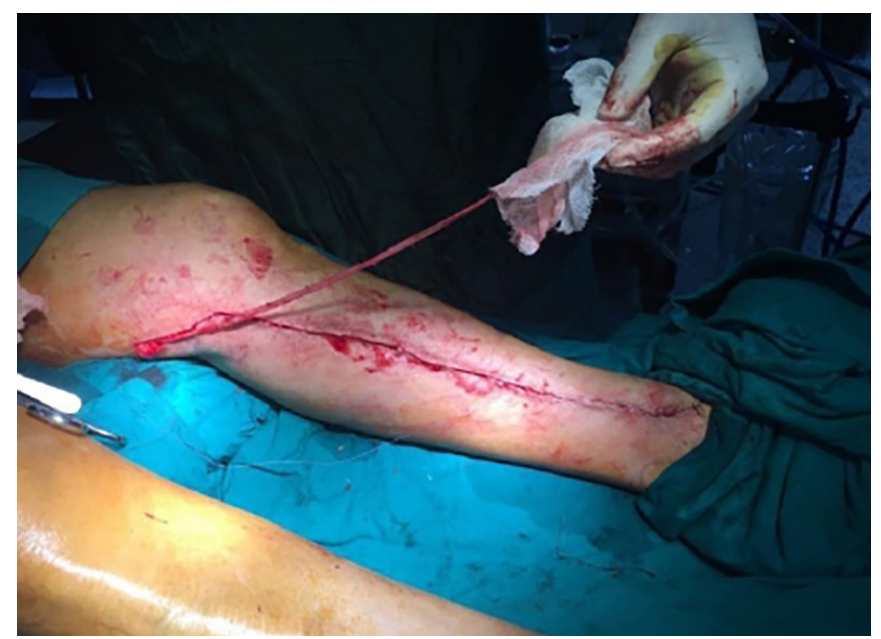

Figure 5. Long incision for $\mathrm{OVH}$
15 cases. Several disposable systems are available, and most frequently used is VirtuoSaph by Terumo. The harvested vein was gently distended with low pressure heparinized saline, and the branches were secured with small clips or repaired by 7-0 Prolene sutures, if needed. The wound was closed at the end of procedure after protamine administration. Drains (Radivac) were used only in 25 cases in EVH, when SVG was harvested from the thigh with a continuous oozing field to decrease hematoma formation. Harvesting time was approximately $35-45$ minutes at the start, then reduced to $25-35$ minutes. The leg was then wrapped in an elastic bandage for 48 hours. Pain assessment and wound examination were performed daily in hospital, 2 weeks, and 4 weeks after discharge. (Figure 1) (Figure 2) (Figure 3) (Figure 4)

OVH was obtained by means of dissection with Metzenbaum scissor. The vein branches were clipped or tied with $3 / 0$ silk. Once fully dissected, the vein was removed. The wound was closed without Radivac, with the exception of two cases. (Figure 5)

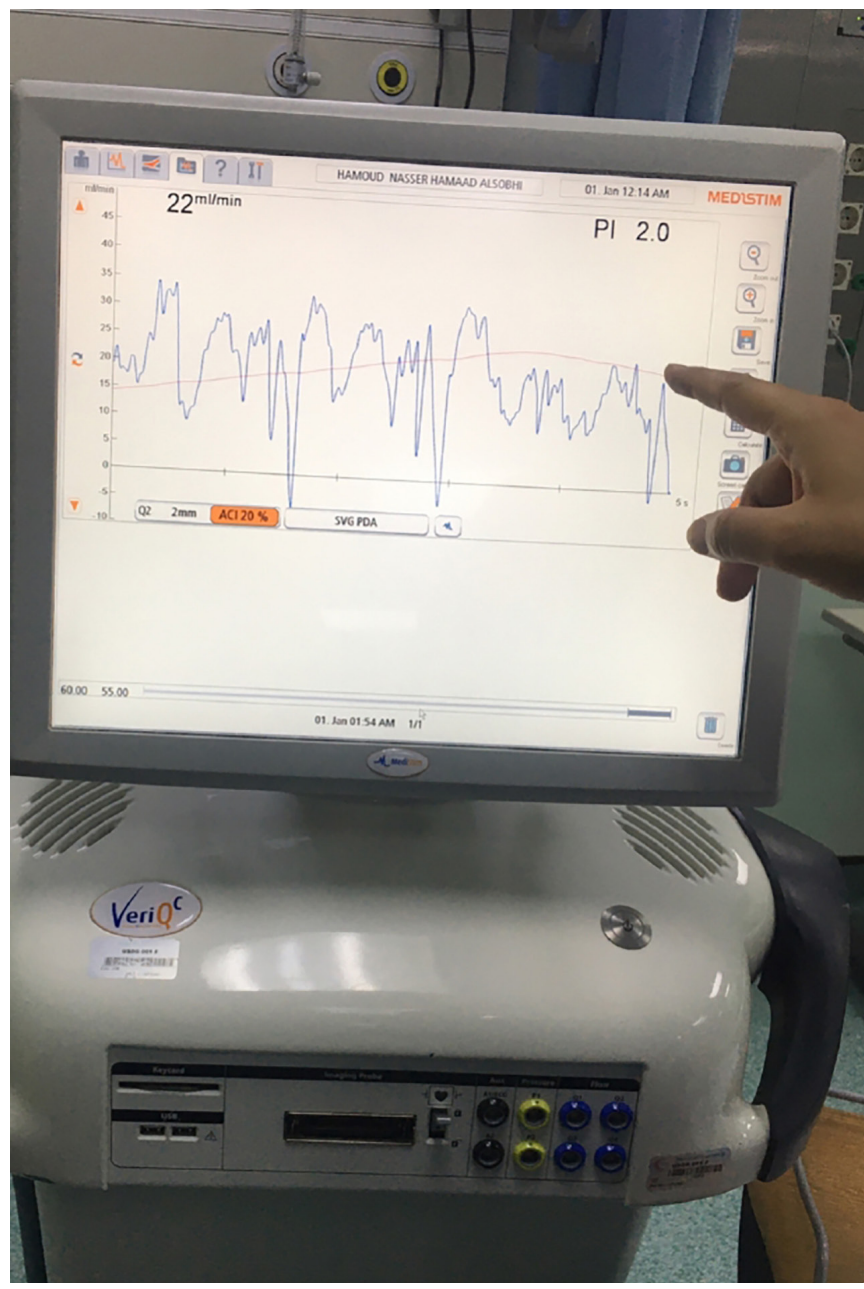

Figure 6. TTFM of GSV grafting intra-operative 


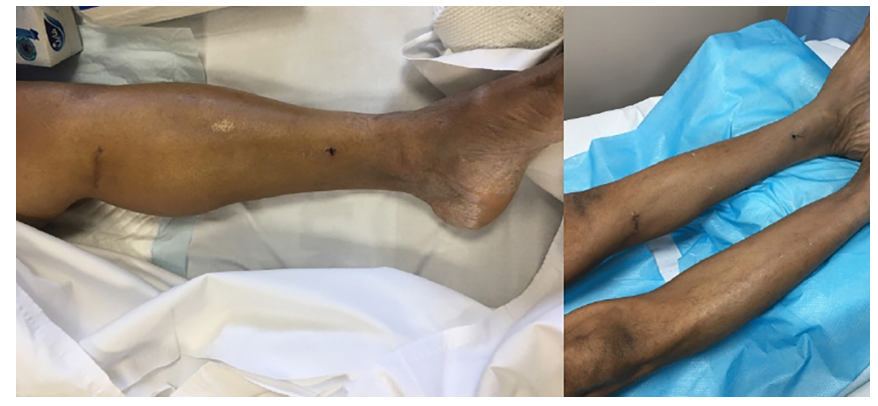

Figure 7. Leg wound follow up of EVH

Pain is assessed by Visual Analog Scale (VAS $=1-10$ ) or by comparison of pain in the leg incision versus sternotomy. In a questionnaire, patients were asked if their leg pain was less than, equivalent to, or more than the pain from the sternotomy. (Diagram 1)

\section{RESULTS}

This prospective, randomized study compared early outcomes of GSV harvesting by endoscopic technique versus open surgical technique. We divided patients into two groups: the EVH group $(N=50)$ and OVH group $(N=50)$. Both groups were similar regarding age, preoperative risk stratification, risks for wound complication, gender, diabetes, obesity, preoperative anemia, hypoalbuminemia, low $\mathrm{EF}<40 \%$, and peripheral vascular disease (PVD). (Table 1) (Table 2)

The primary site of the vein harvested did not influence the incidence of leg wound complications. (Table 3)

$\mathrm{EVH}$ may take longer harvesting time than $\mathrm{OVH}$, however leg wound closure time was longer in $\mathrm{OVH}$. The average time needed for $\mathrm{EVH}$ was $35 \pm 10$ minutes (40-69 minutes). The average length of the harvested vein was $48 \mathrm{~cm}$ (39-55 $\mathrm{cm})$. There was no difference between both groups in CPB time, aortic cross-clamp time, use of IABP, and inotropes. Off-pump CABG (OPCAB) was performed in 15 patients of the EVH group and three patients in the OVH group. The quality of the vein did not appear different between harvest techniques. Transit time Flowmeter routinely was done for all cases. There was no known acute graft closure in either group, and no patient underwent angiography during the follow-up period. Operating room costs were significantly higher when EVH was used compared with OVH. (Figure 6) (Table 4)

Analysis by multiple logistic regressions demonstrated $\mathrm{OVH}$ and uncontrolled DM are risk factors for development of wound complications. Although, obesity and hypoalbuminia were the risk factors for wound complications, their effects were similar in both groups. (Table 5)

The average hospital stay duration for the EVH group was $5.5 \pm 2.4$ days and for the OVH group, it was $9.5 \pm 2.7$ days. Hematoma was detected in two patients of the OVH group, and it was surgically evacuated. We used negative suction drains (Radivac) in 25 cases of EVH without significant

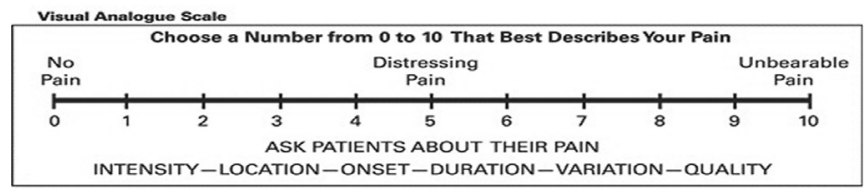

Diagram 1. Wound healing was assessed by recording the presence of postoperative:

(1) Wound drainage (serous, serosanguinous or purulent)

(2) Ecchymosis (hemorrhagic macules in the skin $>2 \mathrm{~cm}$ )

(3) Edema (measured by ankle circumference)

(4) Hematoma (localized blood collection)

(5) Infection (superficial and deep)

(6) Wound dehiscence (need for wound dressings or re-suture)

(7) Length of hospital stay especially when patient discharge was postponed due to leg wound disturbances

Cosmetic result was judged by the patient on the graduated scale:

(1) Unacceptable

(2) Not satisfied

(3) Satisfied

(4) Very satisfied

(5) Extremely satisfied

difference in result. Leg wound infection was more common in OVH than EVH, with significant $P$-value. Infection prolonged hospital stay because culture was taken, waiting for results took time, followed by a full course of antibiotics, and another culture for medical clearance. When leg wound infection was deep, gaped, needed repeated sterile dressing, and sensitive to virulence organism needed IV antibiotics, it affected total hospital stay and cost. Patients were discharged home when the culture was negative, dressing took place once or twice daily, they were on oral antibiotics, or had a nongaped wound. (Figure 7) (Table 6)

There was an improvement from discharge to 4 weeks for pain $(P=0.001)$ and for cosmesis $(P=0.001)$. Patients' rating of leg pain compared with sternotomy pain tended to be lesser in the EVH group. At the end of follow-up period, $99 \%$ of patients were very satisfied with the leg wound in the EVH group as compared with $25 \%$ in the OVH group who were just satisfied.

Mobility was better in the EVH group than the OVH group because of reduced peripheral neuropathy, numbness, pain, knee and ankle movement restriction, edema, and wound infection.

Statistical analysis of continuous variables was performed using Student's t test. Univariate comparison of categorical variables was done using Fisher's exact test and X2 analysis.

\section{DISCUSSION}

GSV remains the most common conduit for CABG, which is the most common operation in cardiac surgery. Surgical site infection (SSI) incidence after GSV harvesting was 3\%-13\% in EVH vs. $12 \%-43 \%$ in OVH [Liliav 2011]. In our study, leg wound infection incidence was $20 \%$ in the OVH group without any recorded infection in the EVH group. 
Table 1. Patient demographics

\begin{tabular}{lccc}
\hline Variable & EVH group $(N=50)$ & OVH group $(N=50)$ & $P$-value \\
\hline Age (years) & $60 \pm 7.5$ & $55 \pm 9.6$ & 0.38 \\
Gender (female) & $13(26 \%)$ & $10(20 \%)$ & 0.21 \\
BMI $\left(\mathrm{kg} / \mathrm{m}^{2}\right)$ & $24.8 \pm 4.5$ & $24.6 \pm 5.2$ & 0.48 \\
HTN & $40(80 \%)$ & $35(70 \%)$ & 0.45 \\
Dyslipidemia & $28(56 \%)$ & $32(64 \%)$ & 0.59 \\
DM & $44(88 \%)$ & $39(78 \%)$ & 0.12 \\
COPD & $1(2 \%)$ & $3(6 \%)$ & 0.14 \\
CKD & $2(4 \%)$ & $4(8 \%)$ & 0.67 \\
PVD & 0 & $1(2 \%)$ & 0.13 \\
Smoking & $15(30 \%)$ & $20(40 \%)$ & 0.87 \\
Hb (gm/dl) & $11.2 \pm 1.7$ & $12.0 \pm 1.6$ & 0.53 \\
EF\% & $46.8 \pm 13.4$ & $45.1 \pm 12.3$ & 0.14 \\
EuroSCORE II \% & $1.7 \pm 1.2$ & $2.1 \pm 1.5$ & 0.56
\end{tabular}

Table 2. Vein harvest site

\begin{tabular}{|c|c|c|c|}
\hline Variable & EVH group $(N=50)$ & \multicolumn{2}{|c|}{ OVH group $(N=50)$} \\
\hline One leg & 50 & & \\
\hline Both legs & 0 & & \\
\hline Leg only & 5 & & \\
\hline Thigh only & 30 & & \\
\hline Extended (leg and thigh) & 15 & & \\
\hline \multicolumn{4}{|c|}{ Table 3. Intra-operative variables } \\
\hline Variable & $\begin{array}{l}\text { EVH group } \\
(N=50)\end{array}$ & $\begin{array}{l}\text { OVH group } \\
(N=50)\end{array}$ & $P$-value \\
\hline Harvested length $(\mathrm{cm})$ & 48.2 & 46 & 0.7 \\
\hline Harvested time (min) & 45.2 & 15.8 & *0.005 \\
\hline Vein preparation time (min) & 1.3 & 1.5 & 0.38 \\
\hline Incision closure time ( $\mathrm{min}$ ) & 3 & 25 & *0.003 \\
\hline No. of grafts & $3.1 \pm 1.1$ & $3.3 \pm 1.0$ & 0.391 \\
\hline No. of SVG bypass & $2.2 \pm 1.0$ & $2.3 \pm 0.9$ & 0.585 \\
\hline
\end{tabular}

Preoperative risk factors of leg wound complications include female gender, DM, obesity, hypoalbuminemia, anemia, PVD, and steroid use. Moreover, tissue trauma and wound closure method may play a role. EVH reduced the effect of diabetes and obesity on the incidence of wound complications. EVH from the thigh may reduce the wound complications of diabetes because of vasculopathy, which is a small vessel disease, affected wound healing more below the knees [Kalra 2016]. In our study, the site of harvesting did not
Table 4. Risk Factor Correlation with Wound Complications

\begin{tabular}{lcc}
\hline Risk factors & Wound complications & P-value \\
\hline Technique & & \\
EVH & $8 \%$ & $* 0.03$ \\
OVH & $66 \%$ & \\
DM & $22 \%$ & $* 0.05$ \\
Non-DM & $9 \%$ & \\
Gender & & \\
Male & $11 \%$ & 0.47 \\
Female & $17 \%$ & \\
Obesity & $20 \%$ & 0.15 \\
Non-obese & $10 \%$ & \\
Hypoalbuminemia & $16 \%$ & 0.47 \\
Non-Hypoalbuminemia & $11 \%$ &
\end{tabular}

affect rate of infection, in spite of the high rate of infection in diabetic patients $-22 \%$ more than non-diabetic $9 \%$.

Compared with OVH, EVH was associated with lower rates of leg wound complications and a shorter hospital stay [Tamura]. Total hospital stay for EVH group was $5.5 \pm 2.4$ days vs. the $\mathrm{OVH}$ group, which was $9.5 \pm 2.7$ days. Moreover, we tried to decrease hospital stay during the COVID-19 pandemic era.

Wound complication can influence the patient's physical activity and may limit his mobility, so it affects the rehabilitation program [Nezafati 2014]. As we know, early mobilization after $\mathrm{CABG}$ is very important for convalescence, and $\mathrm{EVH}$ has a significant early mobilization benefit over OVH $(P=0.02)$.

EVH has been shown to be safe and reproducible with low incidence of leg complications. Patients are satisfied by the 
small incision and absence of leg wound discomfort. As experience grows, a learning curve is required to become familiar with EVH [Raghuram 2007]. Postoperative pain, mobilization, and patient satisfaction were significantly improved in the EVH group.

Concerns exist with regard to thermal spread during diathermic coagulation of side branches, detrimental effects of $\mathrm{CO} 2$ insufflation, and clot formation in the collapsed vein, due to the pressurized working tunnel. There is widespread general acceptance of EVH, doubts about its impact on the integrity, quality of the conduit, and long-term graft patency [Kalra 2016]. In a histological and immunohistochemical evaluation of SVG, EVH showed superiority in endothelial layer preservation, when compared with $\mathrm{OVH}$ [Bouhout 2018]. We did not perform histological examination or vein endoscopy after EVH or coronary angiography during follow-up period, only TTFM was done during surgery.

Leg wound complications can be a major source of postoperative morbidity [Siddiqi 2016]. The best indication for $\mathrm{EVH}$ is patients with increased risk for SSI and for whom cosmetics is a major concern.

Cost may be an important consideration, when choosing EVH. Each endoscopic procedure has a definite added cost, due to the expense of the disposable equipment required along with an initial investment in the non-disposable equipment (monitor, camera, light source, and CO2 insufflator) [Bitondo 2002]. The cost of EVH already is more than OVH, however, the cost of lengthy hospital stay, readmissions, other needed surgical procedures, and antibiotics puts a heavy toll on the health system.

$\mathrm{EVH}$ reduces the incidence of postoperative leg wound complications, especially leg wound infections. Moreover,

Table 5. Measures of wound healing

\begin{tabular}{lccc}
\hline Variable & EVH group $(N=50)$ & OVH group $(N=50)$ & $P$-value \\
\hline Drainage & 0 & 10 & $* 0.001$ \\
Ecchymosis & 1 & 3 & 0.33 \\
Edema & 1 & 4 & 0.2 \\
Hematoma & 0 & 2 & 0.35 \\
Infection & 0 & 10 & $* 0.001$ \\
Dehiscence & 0 & 7 & $* 0.01$ \\
Total & 2 & 36 & $* 0.0001$
\end{tabular}

there are significant differences in postoperative mobility, pain, and satisfaction, favoring EVH. Therefore, the recommendation is routine use of EVH [Kiaii 2002]. Nowadays, surgery has been moved toward less invasive access and minimizing length of incision [Raja 2013]. In the EVH group, patients were very satisfied with the leg wound (99\%), and they experienced significantly less pain and more cosmesis $(P$ $=0.02)$ vs. the OVH group.

The limitations for EVH in CABG increasingly are diminishing [Kirmani 2020]. There is no significant difference in major adverse cardiac events (MACE) among patients who underwent $\mathrm{EVH}$ compared with $\mathrm{OVH}$ for CABG over 5 years of follow up [Zenati 2021]. A well-designed randomized controlled trial (RCT) published in 2019 demonstrated that there was no increase in mortality, myocardial infarction (MI), or repeat revascularisation with EVH. It is a Class-IIa indication in European Association of Cardio-Thoracic Surgery (EACTS) and a Class I indication in International Society of Minimally Invasive Cardiac Surgery (ISMICS) guidelines [Akowuah 2021].

\section{CONCLUSION}

EVH is safe, beneficial, and reliable with better cosmesis, patient satisfaction, and less leg wound complications. It facilitates patient compliance and early mobility. We recommend EVH as a step of MIDCAB and change the old concept of open heart surgery incisions.

\section{LIMITATIONS}

The study includes a small number of patients over a short period. The study compares just leg wound complications between EVH and OVH, without giving any data about histology of the harvested vein and durability of its patency. So, we try to encourage $\mathrm{EVH}$ to minimize terrible leg wound infection in uncontrolled DM that needs hospitalization, IV antibiotics, and vacuum assisted closure (VAC). Microscopic examination is not available after vein harvesting. It is a big issue to do postoperative coronary angiophraphy to assess SVG patency because of the lack of funding for that, and all patients refused to do it. We will try to follow up over 2-5 years and do MDCT for SVG patency.

Table 6. Patient satisfaction

\begin{tabular}{|c|c|c|c|c|c|c|c|}
\hline Satisfaction & \multicolumn{3}{|c|}{ EVH group $(N=50)$} & \multicolumn{4}{|c|}{ OVH group $(N=50)$} \\
\hline Cosmetic satisfaction at discharge & 10 & 9.3 & 1 & 2 & 5.6 & 2 & $* 0.02$ \\
\hline Limited mobility at discharge & 2 & 1.5 & 0.1 & 15 & 8.5 & 0.75 & $* 0.05$ \\
\hline
\end{tabular}




\section{REFERENCES}

Akowuah E, Burns D, Zacharias J, Kirmani BH. 2021. Endoscopic vein harvesting. J Thorac Dis. 13(3): 1899-908.

Bitondo JM, Daggett WM, Torchiana DF, Akins CW, Hilgenberg AD, Vlahakes GJ, et al. 2002. Endoscopic Versus Open Saphenous Vein Harvest: A Comparison of Postoperative Wound Complications. Ann Thorac Surg. 73: 523- 28.

Bouhout I, Ben Ali W, Perrault LP. 2018. The effect of storage solutions on endothelial function and saphenous vein graft patency. Indian J Thorac Cardiovasc Surg. 34 (3):258 - 65.

Kalra S, Aiyer P, Bhardwaj M, Grover V, Gupta VK. 2016. A prospective randomized trial of endoscopic versus open saphenous vein harvesting technique for coronary artery bypass graft surgery. Indian J Thorac Cardiovasc Surg. 32(2):113-19.

Kiaii B, Moon BC, Massel D, Langlois Y, Austin TW, Willoughby A, et al. 2002. A prospective randomized trial of endoscopic versus conventional harvesting of the saphenous vein in coronary artery bypass surgery. J Thorac Cardiovasc Surg. 123: 204-12.

Kirmani BH , Power S , Zacharias J. 2020. Long-term survival after endoscopic vein harvest for coronary artery bypass grafting. Ann R Coll Surg Engl. 102 (6): 422-8.

Liliav B, Yakoub D, Kasabian A. 2011. Case Report; Necrotizing Fasciitis Following Endoscopic Harvesting of the Greater Saphenous Vein for Coronary Artery Bypass Graft. Journal of the Society of Laparoendoscopic Surgeons. 15: 90-95.

Mandiye S, Yadav A, Pathak S, Kanhere VM, Pande H. 2007. Extended length endoscopic harvest of the great saphenous vein for coronary artery bypass grafting. Indian J Thorac Cardiovasc Surg. 23: 125-27.
Moshkovitz Y, Raanani E. 2016. The art of saphenous vein grafting and patency maintenance. J Thorac Cardiovasc Surg. 151: 300-2.

Nezafati MH, Nezafati P, Amoueian S, Attaranzadeh A, Rahimi HR. 2014. Immunohistochemistry comparing endoscopic vein harvesting vs. open vein harvesting on saphenous vein endothelium. J Cardiothorac Surg. 9:101-8.

Raja SG, Sarang Z. 2013. Endoscopic vein harvesting: technique, outcomes, concerns and controversies. J Thorac Disease. 5(6): 630- 37.

Raghuram AR, John EV, Kumar S, Balamurugan K, Amol D, Murugadoss S, et al. 2007. Early experience of endoscopic vein harvesting for CABG. Indian J Thorac Cardiovasc Surg. 23: 188-91.

Raza S, Chang C, Deo SV, Sabik JF. 2018. Current role of saphenous vein graft in coronary artery bypass grafting. Indian J Thorac Cardiovasc Surg. 34:245-50.

Siddiqi MS. 2016. Saphenous vein harvest wound complications: risk factors, identification, prevention, and management. Chronic Wound Care Management and Research. 3: 147-56.

Tamura K, Maruyama T, Sakurai S. The back-approach technique of endoscopic saphenous vein harvesting in coronary artery bypass grafting. Indian J Thorac Cardiovasc Surg.

Zenati MA, Bhatt DL, Stock EM, Hattler B, Wagner TH, et al. 2021. Intermediate-Term Outcomes of Endoscopic or Open Vein Harvesting for Coronary Artery Bypass Grafting The REGROUP Randomized Clinical Trial. JAMA Netw Open. 4(3):e211439.

Zenati MA, Shroyer AL, Collins JF, Hattler B, Ota T, Almassi GH, et al. 2011. Impact of endoscopic versus open saphenous vein harvest technique on late coronary artery bypass grafting patient outcomes in the ROOBY (Randomized On/Off Bypass) Trial. J Thorac Cardiovasc Surg. $141: 338-44$. 